\title{
Comunicación
}

\section{Tratamiento quirúrgico en un bovino con síndrome de indigestión vagal: reporte de caso}

\author{
Surgical treatment in a bovine with vagal indigestion syndrome: case report \\ Cristina García B. ${ }^{1}$, Rocío Sandoval M. ${ }^{1}$, Diego Allcahuamán M. ${ }^{1}$, \\ Alfredo Delgado C. .,2 $^{1}$
}

\section{Resumen}

Se presenta el caso de un bovino hembra de raza Holstein de primer parto, gestante, con un cuadro de distensión abdominal, decaimiento, disminución en la producción de leche. En el examen clínico se observó hipermotilidad ruminal, tanto en la inspección regional como a la auscultación del rumen. En la punción exploratoria en la fosa paralumbar izquierda se observó salida de burbujas, confirmando la presencia de timpanismo de tipo espumoso en el rumen. Los signos clínicos evidenciaron un cuadro compatible con el síndrome de indigestión vagal. Se practicó una laparotomía exploratoria para la confirmación del diagnóstico y la evaluación del compromiso del órgano, y como medida terapéutica una rumenotomía para retirar la mayor cantidad de contenido ruminal. Luego de la intervención quirúrgica se restablecieron las constantes fisiológicas. El animal mostró notable mejoría y culminó la gestación con éxito.

Palabras clave: bovino; hipermotilidad; timpanismo espumoso; indigestión vagal; rumenotomía

\section{AbSTRaCT}

It is reported the case of a pregnant Holstein cow with abdominal distension, depression, decrease in milk production. In the clinical examination ruminal hypermotility was observed, both in the regional inspection and in the auscultation of the rumen. In the exploratory puncture in the left paralumbar fossa, bubbles were observed, confirming the

\footnotetext{
${ }^{1}$ Clínica de Animales Mayores, Facultad de Medicina Veterinaria - Universidad Nacional Mayor de San Marcos, Lima, Perú

${ }^{2}$ E-mail: aldelgadoc@gmail.com
}

Recibido: 6 de junio de 2018

Aceptado para publicación: 17 de octubre de 2018 
presence of foamy tympanism in the rumen. The clinical signs showed a picture compatible with the vagal indigestion syndrome. An exploratory laparotomy was performed to confirm the diagnosis and evaluation of the damage of the organ, and as a therapeutic measure, a rumenotomy was conducted to remove most of the ruminal content. After the surgical intervention, the physiological constants were restored. The animal showed remarkable improvement and ended the pregnancy successfully.

Key words: bovine; hypermotility; frothy bloat; vagal indigestion; rumenotomy

\section{INTRODUCCIÓN}

La indigestión vagal (o síndrome de Hoflund) es un trastorno caracterizado por alteración motora y funcional de los preestómagos en rumiantes adultos, y está asociada a patologías que puedan interferir con el normal recorrido del nervio vago ( $\mathrm{X}$ par craneal), como la reticuloperitonitis a cuerpo extraño (RPCE), abscesos hepáticos y mediastínicos, leucosis, tuberculosis, linfosarcoma, etc. (Scott et al., 2011; Belo Reis et al., 2016). Este síndrome, que se presenta principalmente en bovinos, también ha sido reportado en ovinos (Constable, 2018).

La etiología de este trastorno no se encuentra del todo esclarecida, pero se sugiere que el deterioro mecánico de la motilidad reticular conduce a la falla en el paso normal de la ingesta a través del orificio retículoomasal y el píloro, generando distención abdominal (Eddy, 2004; Scott et al., 2011). Basados en las clasificaciones previas del síndrome de indigestión vagal (Ferrante y Whitlock, 1981), la Universidad de Cornell, EEUU, propone una clasificación donde el Tipo I involucra fallas en el mecanismo del eructo, el tipo II está relacionado a fallas en el transporte omasal, y el tipo III involucra alteraciones en el normal vaciamiento del abomaso (Fubini et al., 2017).
Los signos clínicos dependen de la ubicación del daño al nervio vago y la consecuente alteración que produce. Generalmente hay disminución de ingesta de alimentos, condición corporal y producción de leche. La temperatura rectal y frecuencia cardiaca suelen encontrarse entre rangos normales, aunque puede presentarse bradicardia. Puede presentarse hipermotilidad del rumen, causando distensión del órgano y aumento de las contracciones ruminales. También puede presentarse atonía ruminal con presencia de timpanismo a gas libre y contenido líquido. Así mismo, pueden presentarse casos de estenosis pilórica, donde el abomaso se impacta y distiende por el acúmulo de alimento (Eddy, 2004; Scott et al., 2011; Fubini et al., 2017).

El diagnóstico se basa en los signos clínicos observados a la exploración física y el descarte de otras enfermedades, considerándose la laparotomía exploratoria (Scott et al., 2011) y la punción exploratoria en la fosa paralumbar izquierda (Rimbaud, 2004) como técnicas imprescindibles para el diagnóstico. No hay pruebas de laboratorio específicas; sin embargo, en el caso de una estenosis pilórica se presenta alcalosis metabólica. El diagnóstico diferencial para la indigestión vagal que cursa con dilatación ruminal evidente incluye preñez gemelar, hidropesía alantoidea, peritonitis localizada, atonía ruminal, indigestión simple, timpanismo ruminal, obstrucción intestinal y ascitis (Eddy, 2004; Scott et al., 2011; Constable, 2018). 

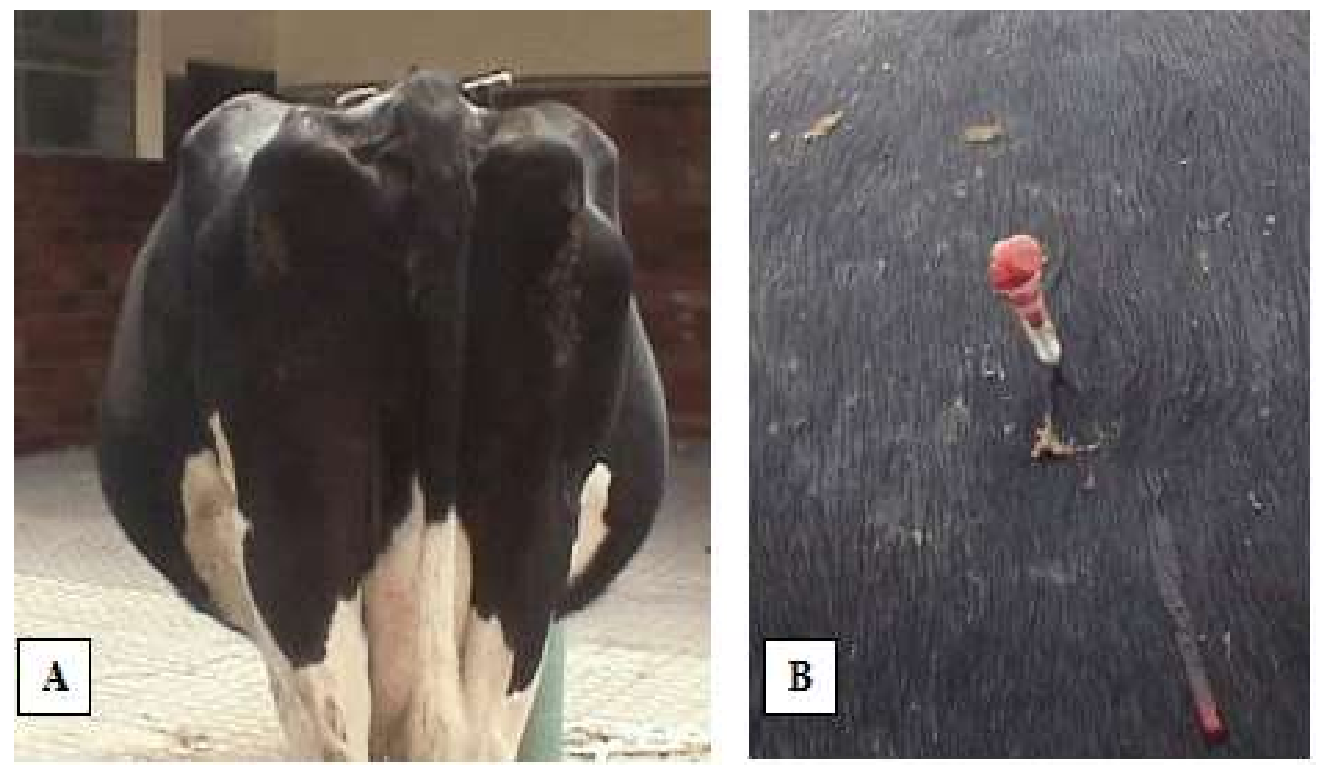

Figura 1. Vista posterior de la vaca (A) y punción exploratoria en la fosa paralumbar izquierda (B)

Por lo general, la indigestión vagal no tiene buen pronóstico, donde menos del $30 \%$ de los casos logran una recuperación exitosa (Haskell, 2008), recomendándose el descarte del animal afectado (Divers y Peak, 2008). Sin embargo, mucho depende de la causa primaria del daño al nervio vago, teniendo mayores posibilidades de recuperación aquellos casos que cursan con hipermotilidad ruminal (Scott et al., 2011). Si bien la laparotomía exploratoria y rumenotomía poseen fines diagnósticos (Divers y Peak, 2008; Foster, 2017), la primera permite detectar y liberar adherencias en la pared abdominal (Eddy, 2004; Haskell, 2008) y la rumenotomía permite el vaciamiento del contenido ruminoreticular (Eddy, 2004; Divers y Peak, 2008). Este reporte presenta un cuadro de hipermotilidad ruminal con evaluación del desempeño del tratamiento quirúrgico.

\section{Caso Clínico}

Un bovino hembra de raza Holstein, de primer parto y $543 \mathrm{~kg}$ de peso fue ingresado a la Clínica de Animales Mayores de la Facultad de Medicina Veterinaria de la Univer- sidad Nacional Mayor de San Marcos por presentar un cuadro de decaimiento, distensión abdominal, disminución en la producción de leche. Datos adicionales de la anamnesis indicaron tratamientos a base de carminativos sin resultados aparentes.

A la inspección se observó una distensión abdominal bilateral (Figura 1), con predominio en el cuadrante ventral derecho y dorsal izquierdo (contorno abdominal de peramanzana) (Smith, 2015), movimientos ruminales evidentes $\mathrm{y}$, aparentemente, incrementados en frecuencia, además de una condición corporal de 2.5/5. La respiración era superficial y con incremento de su frecuencia.

Al examen físico, el paciente presentó un grado de deshidratación de 5\%, temperatura corporal normal $\left(38.2^{\circ} \mathrm{C}\right)$. A la auscultación del rumen, los movimientos ruminales (MR) se encontraban incrementados en frecuencia ( $5 \mathrm{MR} /$ minuto), con amplitud y fuerza disminuidas. Por otro lado, dio negativo a las pruebas estándar de RPCE y desplazamiento de abomaso a la izquierda (Smith, 


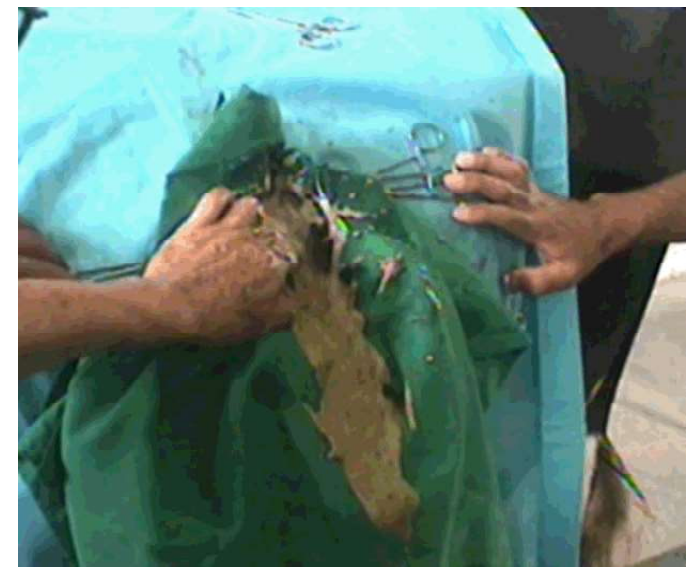

Figura 2. Exploración del rumen y salida del contenido espumoso durante la rumenotomía de una vaca con diagnóstico clínico de indigestión vagal

2015). La frecuencia cardiaca (FC) y la respiratoria (FR) se encontraban incrementadas (FC: 92 lpm; FR: 40 rpm). A la palpación rectal se encontró una ligera disminución del contenido de heces, detectándose también la evidente distención e hipermotilidad ruminal. Por otro lado, el animal dio positivo al diagnóstico de gestación, compatible con preñez de cinco meses.

Se realizó una punción exploratoria a nivel de la fosa paralumbar izquierda con aguja N. ${ }^{\circ} 16$, observándose la salida de algunas burbujas obstruyéndose rápidamente, lo que corroboró la presencia de timpanismo de tipo espumoso en el rumen. En base a los signos y al examen físico, el diagnóstico clínico fue de indigestión vagal, sugiriéndole al propietario realizar una laparotomía exploratoria y rumenotomía.

La laparotomía exploratoria y rumenotomía se realizó con el animal en estación y con anestesia por bloqueo, empleando la técnica de Farquharson (Mansour et al 2018). Se aplicó $10 \mathrm{ml}$ de lidocaína al $2 \%$ en cada punto de bloqueo. Luego de la preparación del campo quirúrgico se realizó una incisión sobre la piel a nivel de la fosa paralumbar izquierda, de aproximadamente $30 \mathrm{~cm}$ de longitud. A continuación, se realizó la disección de los planos subcutáneo y muscular, y mediante una incisión se ingresó a la cavidad abdominal (Figura 2). En la exploración de la cavidad abdominal, se encontraron adherencias de cierta antigüedad en la pared derecha del abdomen, lo que confirma el compromiso del nervio vago. Se observó el rumen con una evidente distención, reconfirmando la hipermotilidad.

Para realizar la rumenotomía (Hendrickson y Baird, 2013) se colocaron dos pinzas Backhaus (Slatter, 2003) en la pared del rumen, sin penetrar a la cavidad, con el fin de sostener la sección del órgano a ser incidida. Se procedió a colocar campos de tercer orden para aislar la cavidad del contenido ruminal que pueda ser expulsado durante el procedimiento. Se realizó una incisión en la pared ruminal de cerca de $20 \mathrm{~cm}$ de largo, resultando en la expulsión violenta del contenido debido a la gran presión intraabdominal. El contenido era líquido espumoso de color verde amarillento. Tras vaciar gran parte del contenido ruminal se realizó la exploración del rumen y retículo, retirándose un cuerpo extraño punzante insertado en la pared reticular lateral derecha, y otros más sueltos sobre el piso del retículo. Luego se hizo tracción de la pared reticular del lado derecho con la intención de liberar las adherencias rumenoreticulares de la pared abdominal derecha. Finalmente, se procedió a cerrar el rumen con un patrón Cushing doble (Slatter, 2003) y la pared abdominal en dos planos con sutura simple continua; en ambos casos se empleó material absorbible (ácido poliglicólico N. ${ }^{\circ} 2$ ). La piel fue suturada con un patrón Reverdin (Slatter, 2003) usando nylon de $0.4 \mathrm{~mm}$.

Finalizado el procedimiento quirúrgico el animal se mostró alerta y se le ofreció alimento y agua ad libitum. Al examen físico, los MR se habían reducido en frecuencia (4 MR en $5 \mathrm{~min}$ ), pero incrementado su fuerza y amplitud. La temperatura corporal se mantuvo normal $\left(39.1^{\circ} \mathrm{C}\right)$. El animal recibió 
antibioterapia posoperatoria con una suspensión a base de penicilina y estreptomicina ( 15 $\mathrm{mg} / \mathrm{kg}$ ) vía intramuscular, cada 12 horas por tres días. Además, se le administró ketoprofeno $(2.5 \mathrm{mg} / \mathrm{kg})$ por el mismo periodo.

A las 24 horas de la cirugía el animal se encontraba alerta y el consumo de alimento se incrementó. Al examen físico la temperatura corporal se encontraba normal $\left(38.6^{\circ} \mathrm{C}\right)$, con 70 latidos por minuto y 25 respiraciones por minuto. Los movimientos ruminales se mantuvieron en $4 \mathrm{MR}$ en $5 \mathrm{~min}$. Las constantes se mantuvieron a lo largo del periodo de internamiento, y tras 15 días de observación fue dado de alta. Se realizó el seguimiento correspondiente del paciente encontrando que el animal mejoró su condición corporal y culminó su periodo de gestación con éxito.

\section{Discusión}

La indigestión vagal está asociada a cualquier patología que pueda interferir con el normal recorrido del nervio vago. En el caso descrito, el problema se presentó aparentemente como consecuencia de una RPCE, la cual representa una de las causas comúnmente asociadas a este trastorno (Scott et al., 2011). Una mala discriminación bucal de los alimentos en el bovino lo hacen susceptible a la ingestión de cuerpos extraños punzantes, generando como consecuencia un cuadro de RPCE. En estos casos, la secuela más común es la formación de adherencias que interfieren físicamente con el nervio vago, dando como resultado la disfunción de los preestómagos (Fubini y Ducharme, 2017).

Debido a las medidas de prevención actuales, los casos de RPCE son muy raros; sin embargo, la presentación de este problema sigue siendo común en algunos lugares (Eddy, 2004), sobre todo en ganaderías a pequeña escala, como el establecimiento de donde provenía el animal del presente caso.
Los signos clínicos que presentó el paciente evidenciaron una lesión a nivel de la rama dorsal del nervio vago, lo que corresponde al tipo I de la clasificación de la Universidad de Cornell (Ferrante y Whitlock, 1981), teniendo mayores posibilidades de recuperación (Scott et al., 2011), lo cual aunado a que el animal se encontraba gestando, fueron las razones que justificaron el planteamiento al propietario de la posibilidad del procedimiento quirúrgico.

Los casos de adherencias múltiples tienen mal pronóstico y no se debería continuar con el procedimiento, ya que el trauma generado por la remoción de estas desencadena un proceso inflamatorio que promueve la formación de nuevas adherencias (Stark, 1984). En el presente caso, las adherencias que se detectaron a nivel de la pared abdominal no estaban totalmente consolidadas, lo que permitió su fácil liberación y la decisión de continuar con la rumenotomía. Algunos autores consideran que la rumenotomía y el vaciamiento del rumen pueden proporcionar ventajas terapéuticas (Drivers y Peak, 2008; Foster, 2017). El vaciamiento del rumen restablecería la función de los receptores de presión, alterada por la distención masiva. Además, estos receptores de presión junto con los del retículo promoverían la contracción de los pre-estómagos, siempre y cuando el daño al nervio vago lo permita (Drivers y Peak, 2008).

En el presente caso, las contracciones que presentaba el rumen antes de la apertura eran de poca fuerza o intensidad; sin embargo, la rumenotomía y el vaciamiento del contenido habrían liberado la presión ejercida sobre la pared del órgano, lo cual pudo haber permitido un incremento de la intensidad de las contracciones del órgano. A la evaluación posquirúrgica se habían restablecido las constantes fisiológicas (FC, FR y MR), aparentemente favorecidas por el tratamiento quirúrgico. 
Por lo general, la indigestión vagal no tiene buen pronóstico (Drivers y Peak, 2008); sin embargo, en algunas ocasiones se puede recurrir al tratamiento quirúrgico como alternativa para la resolución a este tipo de trastorno, siempre que se reúnan las condiciones adecuadas, como las mencionadas en el presente caso.

\section{Literatura Citada}

1. Belo Reis A, Anjos H, Chaves CM, Sousa CH, Sarmento JA, da Silva N, Masiero F, et al. 2016. Vagal indigestion in zebu cattle in Brazil. Rev Salud Anim 38: 149-153.

2. Constable PD. 2018. Vagal indigestion syndrome in ruminants. MSD Veterinary Manual [Internet]. Disponible en: https:/ /www.msdvetmanual.com/digestivesystem/diseases-of-the-ruminant-forestomach/vagal-indigestion-syndrome-inruminants

3. Divers TJ, Peak SF. 2008. Rebhun's diseases of dairy cattle. $2^{\text {nd }} e d$. USA: Saunders Elsevier. 686 p.

4. Eddy RG 2004. Alimentary conditions. In: Andrews AH (ed). Bovine medicine. Diseases and husbandry of cattle. $2^{\text {nd }} \mathrm{ed}$. UK: Blackwell Science. p 821-859.

5. Ferrante PL, Whitlock RH. 1981. Chronic vagal indigestion in cattle. Comp Cont Educ 6: 231-237.
6. Foster F. 2017. Disorders of rumen distension and dysmotility. Vet Clin North Am Food Anim Pract 33: 499-512. doi: 10.1016/j.cvfa.2017.06.006

7. Fubini S, Ducharme N. 2017. Farm animal surgery. $2^{\text {nd }}$ ed. USA: Elsevier. 662 p.

8. Haskell SRR. 2008. Blackwell's fiveminute veterinary consult: ruminant. USA: Wiley-Blackwell. 1048 p.

9. Hendrickson DA, Baird AN. 2013. Turner and McIlwraith's techniques in large animal surgery. $4^{\text {th }}$ ed. USA: Wiley Blackwell. $331 \mathrm{p}$.

10. Mansour M, Wilhite R, Rowe J. 2018. Guide to ruminant anatomy: dissection and clinical aspects. USA: Willey Blackwell. 293 p.

11. Rimbaud E. 2004. Semiología, semiotecnia y propedéutica de los bovinos. Nicaragua: Univ. de Ciencias Comerciales. 89 p.

12. Scott PR, Penny CD, Macrae AI. 2011. Cattle medicine. UK: CRC Press. 288 p.

13. Slatter DH. 2003. Textbook of small animal surgery. Vol $1.3^{\text {rd }}$ ed. USA: Elsevier Health Sciences.

14. Smith BP. 2015. Large animal internal medicine. $5^{\text {th }}$ ed. USA: Elsevier Mosby. $1661 \mathrm{p}$.

15. Stark DA. 1984. Illness associated with abdominal adhesions in a cow. Mod Vet Pract 65: 386-368. 